\title{
Criminologie
}

\section{La justice réparatrice en Allemagne}

\section{Frieder Dünkel}

Volume 32, numéro 1, printemps 1999

La justice réparatrice

URI : https://id.erudit.org/iderudit/004718ar

DOI : https://doi.org/10.7202/004718ar

Aller au sommaire du numéro

Éditeur(s)

Les Presses de l'Université de Montréal

ISSN

0316-0041 (imprimé)

1492-1367 (numérique)

Découvrir la revue

Citer cet article

Dünkel, F. (1999). La justice réparatrice en Allemagne. Criminologie, 32(1),

107-132. https://doi.org/10.7202/004718ar

\section{Résumé de l'article}

L'article décrit la mise en place des programmes de médiation en Allemagne. La médiation est d'abord devenue un élément majeur de la justice des mineurs, pour s'appliquer ensuite à la justice criminelle en général. $74 \%$ des départements locaux de protection de la jeunesse ont introduit des programmes de médiation. En Allemagne de l'Est (après la réunification de 1990), les programmes de médiation sont même plus développés que dans les anciens États fédéraux. En Allemagne, l'approche de la justice réparatrice fait désormais partie du système de justice criminelle ; cette approche est gérée par des organismes privés et publics. Dans cet article, l'auteur décrit aussi l'expérience de la justice réparatrice en Europe depuis les dix dernières années. 


\section{La justice réparatrice en Allemagne}

Frieder Dünkel

Professeur de criminologie et de droit criminel

Université de Greifswlad · Allemagne

duenkel@mail.uni-greifswald.de

RÉSUMÉ L'article décrit la mise en place des programmes de médiation en Allemagne. La médiation est d'abord devenue un élément majeur de la justice des mineurs, pour s'appliquer ensuite à la justice criminelle en général. $74 \%$ des départements locaux de protection de la jeunesse ont introduit des programmes de médiation. En Allemagne de l'Est (après la réunification de 1990), les programmes de médiation sont même plus développés que dans les anciens États fédéraux. En Allemagne, l'approche de la justice réparatrice fait désormais partie du système de justice criminelle ; cette approche est gérée par des organismes privés et publics. Dans cet article, l'auteur décrit aussi l'expérience de la justice réparatrice en Europe depuis les dix dernières années.

ABSTRACT The paper describes the introduction of mediation schemes in Germany, which have become a major issue for juvenile justice policy and lately for criminal policy in general. Seventy-four percent (74\%) of all German local youth departments have introduced mediation schemes. The situation in East Germany (after the reunification in 1990) is even better than in the old Federal states. The restorative justice approach in Germany is integrated in the juvenile justice system and organized by private and state institutions. The author outlines the European experiences with restorative justice during the last decade.

Criminologie, vol. 32, $n^{\circ} 1$ (1999) 


\section{L'émergence de la justice réparatrice : médiation et réparation}

En Allemagne, des éléments de justice réparatrice furent d'abord introduits dans le cadre de la justice des mineurs touchant les jeunes délinquants de 14 à 21 ans $^{1}$. Une nouvelle loi sur la justice des mineurs fut promulguée en Allemagne à la fin de 1990. En plus de nouvelles sanctions communautaires, cette loi établissait un cadre légal dans lequel la médiation jouait le rôle de sanction judiciaire à caractère éducatif et de stratégie de poursuite alternative (diversion).

Les textes justifiant l'adoption de la nouvelle loi faisaient mention des expériences favorables découlant des projets-pilotes lancés depuis 1985. Ces projets avaient entraîné une prise de conscience accrue de la situation spéciale des victimes et « résolu le conflit opposant contrevenant et victime à la suite de l'acte criminel d'une façon plus opportune et avec plus de succès [...] que les sanctions traditionnelles ne l'avaient fait par le passé » (Bundesratsdrucksache, $n^{\circ} 464 / 89$, p. 44). Le législateur centrait alors tout son intérêt sur la réparation lorsqu'il s'agissait de crimes commis par des jeunes âgés de 14 à 21 ans. Cette tendance était assez surprenante pour l'époque puisque les expériences menées dans le cadre de projets de médiation étaient encore assez récentes. Les premiers projets-pilotes concernant la justice des mineurs débutèrent en 1985. À la fin des années 1980 , environ vingt projets majeurs existaient, incluant quelques projets ayant trait aux adultes (Bannenberg, 1993 ; Hering et Rössner, 1993 ; Marks et Meyer, 1994 ; Pfeiffer, 1997 ; Dölling et al., 1998).

La médiation couvre un large éventail de mesures individuelles (spécialement depuis la réforme légale de 1990). Au début des années 1990, 224 institutions interrogées dans le cadre d'une enquête nationale (des anciens États fédéraux) indiquaient qu'elles avaient déjà mis en place ou qu'elles projetaient d'établir concrètement des programmes de médiation (Schreckling et al., 1991). 60 \% des départements de protection de la jeunesse ont par la suite mis en place des programmes de médiation. $85 \%$ des institutions travaillaient avec des mineurs ou de jeunes adultes. Un nombre de plus en plus grand de projets concernait aussi les adultes de plus de 21 ans. Dans une enquête pour l'année 1995, Wandrey et Weitekamp ont recensé 368 projets de médiation, c'est-àdire une augmentation de $68 \%$ depuis 1992. Les projets augmentèrent encore d'environ 2100 à 9000 en 1995, c'est-à-dire de $78 \%$ sur une

I. Pour avoir un aperçu du système de justice des mineurs en Allemagne, voir Kaiser, 1994. 
période de trois ans. Cependant, les auteurs mentionnent que la plupart des projets effectués dans le champ de la justice des mineurs font rarement appel à la médiation et ne l'emploient que comme une « approche ad hoc», secondaire par rapport à d'autres mesures éducatives (voir Wandrey et Weitekamp, in Dölling et al., 1998 : 130 ss.). Parmi tous les programmes de médiation, les projets relatifs aux adultes de plus de 21 ans augmentèrent de $28 \%$.

Récemment, une autre enquête, dirigée par le département de Criminologie de l'Université de Greifswald à la demande du ministère fédéral de la Justice, a révélé qu'en 1993-1994, la médiation était désormais disponible dans tous les anciens États fédéraux (et aussi dans les nouveaux États depuis la réunification de l'Allemagne). 70 \% des départements de protection de la jeunesse interrogés dans les anciens États fédéraux et $88 \%$ dans les nouveaux rapportaient que la médiation était offerte par des travailleurs sociaux faisant partie des départements de protection de la jeunesse ou par des services privés d'aide aux jeunes. Ce choix était offert en tant que stratégie avant la sanction pénale (diversion) dans environ trois quarts de tous les districts de protection de la jeunesse $(74 \%)$. Presque la moitié des départements de protection de la jeunesse $(48 \%)$ offraient la médiation dans leurs propres services. Un $11 \%$ additionnel fournissait ce service conjointement ou aux côtés d'un service privé et, dans $14,5 \%$ des cas, la médiation était prise en charge exclusivement par un service privé (une comparaison régionale de la disponibilité des programmes de médiation est mentionnée ci-après, voir Dünkel, Kirstein et Geng, 1998).

Actuellement, le nombre de projets de médiation augmente encore. Une récente enquête dans les nouveaux États fédéraux a recensé 128 projets en 1997 alors qu'en 1994, «seulement » 48 avaient été mis en place (voir Steffens, sous presse). Ainsi, Wandrey et Weitekamp ont-ils raison d'affirmer que la médiation en Allemagne est encore en pleine croissance. Cependant, le danger est grand que la médiation ne joue que le rôle de sanction éducative/réhabilitative dans le système traditionnel de justice pour les jeunes et les adultes contrevenants plutôt que d'être un pas vers l'établissement d'une réelle justice réparatrice.

Les études empiriques ont confirmé à plusieurs reprises l'acceptation largement répandue de la médiation en Allemagne (sur l'esprit de la réparation, voir, entre autres, Sessar et al., 1986 ; Voss, 1989 : 43 ss. ; Sessar, 1992 ; Pfeiffer, 1994 ; Kilchling, 1995 ; Dölling et Henninger, dans Dölling et al., 1998 : 360 ss.). Cette attitude favorable se retrouve 
tant dans les factions politiques plutôt conservatrices (soulignant les intérêts de la victime) que dans les groupes de tendance abolitionniste (considérant la médiation comme une opportunité pour reprivatiser les conflits ; Pfeiffer, 1992 : 338, 345).

\section{Le cadre légal de la médiation}

Le droit allemand actuel — spécialement pour les mineurs — offre beaucoup de possibilités pour faciliter la réparation des torts et la médiation. Le ministère public siégeant au tribunal des mineurs peut abandonner les poursuites si des mesures de réforme ont déjà été appliquées ou introduites ( $§ § 45$ II JGG, Loi sur la justice des mineurs). La loi de 1990 met explicitement sur un pied d'égalité la médiation et ces mesures de réforme. De manière significative, le législateur reconnaît déjà les efforts sincères faits par les mineurs pour résoudre les conflits ou pour réparer leurs torts. Ces mesures protègent les mineurs et les jeunes adultes contrevenants si la victime refuse de coopérer. La réussite d'une démarche de réparation entraîne souvent un acquittement pour cause de "culpabilité réduite " (conformément à $\S \S 45 \mathrm{IJGG}$ ou $\S \S 153 \mathrm{StPO}$ dans le droit criminel pour adultes). Le ministère public du tribunal de la jeunesse, comme les juges siégeant à ce tribunal, peut abandonner les poursuites compte tenu des efforts de médiation manifestés par les jeunes délinquants. Des particularités propres à la législation allemande concernent aussi la restitution de biens matériels perdus, la médiation et la possibilité pour les juges d'ordonner le recours à la médiation (en tant que sanction indépendante ; voir $\S \S 15,10$ JGG). En 1994 cependant, le législateur introduisit la clause 46a dans le Droit pénal (StGB) qui stipule que si le contrevenant, " par les efforts qu'il a faits pour en arriver à un règlement du conflit avec la partie lésée, a dédommagé entièrement ou partiellement la victime ou du moins a sincèrement essayé de réparer son acte, ou si la réparation exige un service ou des sacrifices personnels trop importants de sa part pour satisfaire la victime, le tribunal peut [...] réduire la peine » ou, dans le cas de peine d'un an et moins d'emprisonnement, la supprimer entièrement. La justice des mineurs, comme celle des adultes, prévoit que la réparation des torts s'accompagne parallèlement d'une suspension de l'incarcération dans une maison de détention provisoire ou en prison (les mêmes conditions s'appliquent pour la mise en probation ; pour un résumé, voir Dünkel et Rössner, 1989 ; Rössner et Krauss, 1998). Les 
clauses légales précédentes ont été appliquées dans toute l'Allemagne depuis la réunification en octobre 1990. Le recours à des comités d'arbitrage spécialisés dans la résolution de conflits internes ou de conflits de voisinage, qui prévalait en République démocratique allemande, n'était malheureusement plus possible ${ }^{3}$. La réparation en tant que sanction infligée par le tribunal des mineurs reste une pratique assez limitée : en 1996, 1,8 \% des sanctions imposées aux mineurs emprisonnés comprenait une ordonnance de réparation (voir Strafverfolgungsstatistik, 1996).

Faire de la médiation une sanction applicable par le tribunal des mineurs (voir $\S \S 10$ I n $^{\circ} 7 \mathrm{JGG}$ ) fut à juste titre critiqué comme violant le principe suivant lequel les efforts de médiation devaient s'effectuer sur une base volontaire. En pratique, la médiation en tant que directive de type éducatif imposée par la cour des mineurs n'est pas utilisée (voir Rössner et Klaus, 1998 : 115), parce que les cas poursuivis sont traités de façon informelle (diversion dans le sens de la loi $\S \S 45$ II JGG, voir ci-dessus) devant un tribunal et ne s'inscrivent donc pas dans les procédures formelles des tribunaux.

\section{Cadre conceptuel des projets de médiation}

Malgré des différences d'organisation, il existe certains points communs entre les projets, spécialement en ce qui concerne les buts et les procédures durant les négociations concrètes. Tous les projets menés en Allemagne fédérale concernent les cas relevant de la justice criminelle ; ils ne concernent que rarement les conflits de voisinage traités au civil, contrairement à ce qui se fait dans les bien connus Neigbbourbood Justice Projects aux États-Unis (voir à ce sujet Dünkel, 1990a). Les projets mettent l'accent sur le règlement du conflit plutôt que sur l'exercice de la justice criminelle. Cette perspective amène à prendre en compte le vécu subjectif de la partie lésée, au lieu de se conformer aux critères judiciaires classiques mettant plutôt l'accent sur la gravité de l'acte et la culpabilité, les délits antérieurs commis par l'accusé et ainsi de suite (Messmer, 1996). La médiation met en lumière les efforts de négociation directe entre le contrevenant et la victime (qui a généralement subi des

3 Voir à ce sujet, la loi du 13 septembre 1990 relative à la cour d'arbitrage de la RDA, qui dissout de tels comités. Les comités d'arbitrage qui étaient censés remplacer ce système n'eurent guère d'effets significatifs, leur zone d'intervention étant limitée à des cas très insignifiants, voir Sabrotzky (1997). 
dommages). La rencontre entre le contrevenant et la partie lésée ainsi que la démarche de réconciliation arbitrée par un tiers impartial composent la trame principale de la médiation. Il existe cependant d'autres formes disponibles de résolution des conflits (spécialement si la victime ne désire pas de rencontre personnelle mais est plutôt intéressée par une réparation). La médiation poursuit trois objectifs :

1) la réconciliation entre l'agresseur et la victime en vue de régler le conflit causé par l'acte criminel ;

2) la réparation financière ou symbolique par le contrevenant du tort matériel ou non matériel (par exemple de l'argent en réparation de la douleur et de la souffrance);

3) la prise en compte des démarches de réparation en abandonnant les poursuites au criminel ou en réduisant la sanction infligée.

L'étape décisive du processus de résolution de conflit n'est pas tant le résultat final que le processus de médiation qui implique activement le contrevenant et la victime, accordant de ce fait aux personnes une autonomie et un champ d'action qui avaient disparu dans les procédures criminelles classiques.

L'expérience menée en République fédérale allemande montre que le contact établi entre le contrevenant et la victime (sans nécessairement aboutir à une réconciliation qui annule le conflit) peut amoindrir sinon éliminer le désir de la victime que le contrevenant reçoive une peine criminelle. Même la réparation des dommages revêt moins d'importance que les actes symboliques de réconciliation, comme des excuses.

Les formes suivantes de médiation des conflits se sont aussi révélées efficaces :

- conversations entre les deux parties suivies d'excuses ou de paiement pour les pertes matérielles (généralement moins que $150 \$ 4$ );

- services rendus à la partie lésée afin de compenser le tort qui a été fait ;

- services communautaires rendus, payés par un fonds : l'agresseur donne les profits de ce travail à la victime ;

- activités menées en commun par les victimes et les agresseurs ;

- cadeaux en tant que gestes symboliques de réconciliation (Kuhn et al., 1989).

De tels services doivent se limiter exclusivement à la démarche réparatrice et n'ont pas pour fonction d'assurer un suivi sociopédagogique à long

4. Les résultats d'une enquête nationale indiquent que le paiement exigé ne dépassait pas 250 marks pour $54 \%$ des cas (c'est-à-dire environ 150 \$ US), 73\% des cas devaient payer moins de 450 marks (c'est-à-dire 270\$ US). 
terme $e^{5}$. À part la réparation active, le contrevenant ne devrait pas devenir un objet de soins sociopédagogiques» (Kuhn et al., 1989). Cette démarche ne vise pas non plus à prendre en charge la victime ou de lui offrir une thérapie. Le résultat souhaité dans une telle démarche est d'entraîner à long terme une prise de conscience chez le contrevenant (l'éclairant sur l'injustice commise, le dissuadant de commettre à nouveau de tels actes) et de rassurer la victime afin de la soulager de la blessure ou du traumatisme résultant des actes du contrevenant.

\section{Organisation des projets de médiation}

En Allemagne, la médiation est organisée de plusieurs façons. D'après les règles de base, les tribunaux disposent d'une plus grande marge de manœuvre quand ils confient les cas de médiation aux services privés ; les cas confiés aux services d'aide des tribunaux de la jeunesse (c'est-àdire à des travailleurs sociaux rattachés au ministère public) sont traités d'une façon plus conforme aux normes du système judiciaire traditionnel. De plus, les services d'aide des tribunaux de la jeunesse prennent habituellement des mesures plus centrées sur le contrevenant ${ }^{6}$. Un troisième modèle d'organisation a été développé dans les nouveaux États fédéraux de Brandebourg et de Saxe-Anhalt après la réunification de l'Allemagne. Les ministres de la Justice mirent en place un nouveau service social destiné spécialement à la médiation (voir Steffens, sous presse). Ces services publics indépendants peuvent plus facilement maintenir l'équilibre entre la victime et l'agresseur que les travailleurs sociaux qui traditionnellement travaillent surtout avec les contrevenants. Comme ces services sont subventionnés par l'État, ils n'ont pas les problèmes que connaissent les organisations privées pour trouver des fonds pour leur personnel. Les services d'aide officiels et les services privés different aussi sur d'autres points. Beaucoup de projets menés par les services rattachés aux tribunaux de la jeunesse assurent la médiation

5. Voir Schreckling et al., (1991: 20) en ce qui concerne les «interventions sociopédagogique brèves »; pour les bases méthodologiques et théoriques, voir spécialement Kawamura et Schreckling (1990) et Messmer (1996).

6. $\S \S 38$ II JGG : «Les responsables du service d'aide considèrent qu'ils ont pour fonction de transformer, de socialiser et de prendre soin des jeunes contrevenants. Ils aident les autorités à enquêter sur la personnalité, le développement et l'environnement de l'accusé et formulent les mesures à prendre. Si aucun agent de probation ne prend en charge le jeune, ils s'assurent que ce dernier se plie aux instructions et aux ordres [...]». 
par le biais de travailleurs sociaux qui remplissent d'autres tâches, par exemple l'organisation des travaux communautaires, la supervision et l'organisation de programmes d'acquisition de compétences psychosociales pour les contrevenants, etc. Ces pédagogues ou travailleurs sociaux ne sont souvent pas spécialisés dans le domaine de la médiation (voir Dünkel, Kirstein et Geng, 1998). Cependant, de plus en plus de projets, particulièrement ceux qui sont menés par des organisations privées ou des services sociaux spécialisés, disposent d'un personnel mieux entraîné et plus qualifiét. Tous ces travailleurs n'exercent pas non plus leurs activités dans le seul champ de la médiation. Wandrey et Weitekamp (dans Dölling et al., 1998 : 121) définissent leur travail comme "partiellement spécialisé ». On prend de plus en plus conscience que la procédure centrée sur le contrevenant, qui est en cours traditionnellement dans les services d'assistance des tribunaux de la jeunesse, ne peut satisfaire aux exigences requises de la part d'un négociateur impartial ${ }^{8}$.

En conséquence, beaucoup de projets effectués au sein des services d'aide des tribunaux de la jeunesse (départements locaux de la jeunesse) emploient maintenant du personnel plus formé et plus concentré sur le travail de médiation (voir Hartmann, 1995 ; Dölling et al., 1998). Quand on désire régler un cas de façon plus efficace, on préfere souvent assigner la médiation à des services privés. Néanmoins, les projets gérés par des services privés sont menés en étroite collaboration avec les tribunaux et concernent des cas qui ont été référés par le ministère public ou par le service d'aide du tribunal de la jeunesse. Cette procédure est spécialement conforme aux valeurs sous-tendues dans des projets qui privilégient un règlement privé et extrajudiciaire des conflits (Kuhn et al., 1989). Ajoutons ici qu'une formation spécialisée pour les médiateurs ainsi que l'élaboration de lignes directrices spécifiques pour le processus de négociation semblent impératives. La Deutsche Bewäbrunggsbilfe (association d'aide à la probation), qui gère une agence spéciale de services, étudie systématiquement le sujet et a développé des modèles de projets de médiation. Comment les coûts liés à la main-d'œuvre et les fonds nécessaires à la réalisation de projets de médiation peuvent-ils être évalués? Les projets menés en Allemagne fédérale montrent qu'un travailleur social peut traiter environ quatre-vingts à

7. Pour un résumé de la recherche concernant certains projets-pilotes dans le domaine de la justice des mineurs et la justice criminelle des adultes, voir Schreckling et al., (1991); Hering et Rössner (1993), Rössner et Bannenberg (1994), Dölling et Stroetzel, dans Dölling et al., (1998: 160-162).

8. Sur les problèmes pratiques relatifs à la médiation, voir Hassemer, dans Dölling et al., 1998. 
cent cas par année, ce qui suppose des entrevues avec 150 contrevenants ou victimes. La médiation coûte donc assez cher en termes de main-d'œuvre et de temps. Bien sûr, en maintenant l'opération dans le cadre du programme d'assistance offert par le tribunal de la jeunesse, on n'entraîne pas de frais supplémentaires. En tenant compte de la chute importante du recours à l'aide fournie aux jeunes par les tribunaux à cause de la baisse du taux de natalité et en raison de la baisse absolue et relative de la délinquance juvénile depuis les années 1980, spécialement pour les crimes graves (qui faisaient particulièrement l'objet de l'aide des tribunaux), on a pu disposer de plus de main-d'œuvre pour la médiation. Cependant, avec, d'une part, l'augmentation du taux de criminalité depuis la fin des années 1980, et, d'autre part, la réduction des budgets sociaux, les personnes travaillant dans les services d'aide juridique ont rencontré des problèmes. Certains changements structurels dans le cadre des départements locaux de la jeunesse ont entravé le bon fonctionnement des programmes de médiation, des cours de formation sociale et d'autres sanctions communautaires de type sociopédagogique prévues par la Loi sur la justice des mineurs. Les projets menés par les services privés rencontrent aussi des problèmes de financement (voir Dünkel et al., 1998). Cependant, en général, les municipalités et les tribunaux (qui allouent les profits tirés des amendes à de telles organisations à but non lucratif) ou d'autres services privés (églises, fondations privées, etc.) fournissent des ressources. Malgré tout, le manque d'argent est devenu récemment plus préoccupant. La récession générale a réduit les moyens financiers des villes et des communautés, de même que les ressources des programmes d'aide à la main-d'œuvre. Certains projets ont souligné la nécessité de mettre en place un fond destiné aux victimes. De tels fonds sont constitués du produit des amendes. Des contrevenants sans ressources sont payés à l'aide de ce fonds pour rendre des services communautaires et sont donc aptes à réparer les torts qu' ils ont commis en donnant leur salaire aux victimes. Les mineurs (qui sont plus souvent sans argent) sont particulièrement intéressés par ce type d'option : en ayant accès à de tels fonds, ils évitent de rester en dette vis-à-vis de la partie lésée.

\section{Nouveaux cas et critères d'attribution}

Comme nous le mentionnions précédemment, tous les projets de médiation menés en Allemagne fédérale - spécialement ceux qui ne sont pas traités dans le cadre des tribunaux (assistance par le tribunal de la 
jeunesse) - supposent une étroite collaboration avec le ministère public du tribunal de la jeunesse et avec les tribunaux eux-mêmes. En conséquence, la plupart des cas sont présélectionnés par des travailleurs sociaux employés par le service d'assistance du tribunal de la jeunesse ou par le ministère public. Quelquefois, la police aide à sélectionner les cas devant faire l'objet d'un suivi.

Le ministère public du tribunal de la jeunesse reste cependant le principal responsable de la sélection. Les projets sélectionnés, comme cela se passe à Cologne et Reutlingen, permettent d'assurer la médiation au sein des tribunaux avant ou même pendant le procès. À Cologne, même le tribunal de la jeunesse peut occasionnellement veiller à trouver un arrangement entre les parties pendant le procès (voir Schreckling et al., 1991). En Allemagne fédérale, les cas pouvant faire l'objet de médiation doivent obéir aux critères de sélection suivants :

- Obtention préalable des aveux : On exige des aveux du contrevenant ainsi que des éclaircissements sur les circonstances de son acte. Ces informations garantissent que la médiation ne porte pas préjudice au droit à la défense de l'accusé ou aux principes traditionnels d'un jugement équitable (par exemple le respect de la présomption d'innocence).

- «Clause des délits mineurs»: La médiation est considérée uniquement dans les cas assez graves pour justifier une poursuite $(\S \S 45$ I JGG ou $\S \S 153$ StPO). Ce critère empêche l'extension ou l'intensification du contrôle social dans le domaine de la justice des mineurs.

- Présence d'une victime : La présence d'une victime qui a subi un dommage personnel est généralement requise. La médiation, qui se fonde principalement sur la rencontre personnelle ou la confrontation entre le mineur et la victime, ne semble pas s'appliquer aux cas de vol dans les magasins, de fraude et de violation du code de la route n'ayant pas entraîné de dégâts matériels ou de blessures. À l'occasion, les propriétaires de petits magasins ou d'institutions (garderies et autres services au public) peuvent être impliqués dans un échange contrevenant-victime.

- Le volontariat : L'accord du contrevenant et de la victime - sans aucune pression extérieure - est un point crucial. Cependant, le concept de bon vouloir amène des interrogations si l'alternative à la médiation proposée à l'accusé est un jugement au criminel. La bonne volonté n'est pas alors définie en termes positifs. La démarche de médiation ne devrait être soumise à aucune forme de pression extérieure.

Les projets menés en Allemagne fédérale ont aussi démontré qu'il ne faut pas exclure plus spécifiquement certains délits ; réduire l'exercice de 
la médiation à de petits délits ou à des infractions moins graves (excluant donc les actes criminels) est particulièrement inapproprié. Par exemple, certains crimes qui sont considérés comme des actes criminels, tels que le vol qualifié, le vol à main armée et même des délits sérieux d'ordre sexuel, peuvent faire l'objet de médiation. De tels cas composent 5 à $10 \%$ de toutes les affaires traitées dans le domaine de la justice des mineurs ${ }^{9}$. La médiation est aussi utile pour les cas de récidive. En septembre 1989 à Göttingen, la $21^{\mathrm{e}}$ assemblée du tribunal allemand de la jeunesse a conclu que les expériences pratiques indiquaient que la médiation est de plus en plus utilisée pour des délits plus graves (par exemple dommages physiques sévères, vol avec circonstances aggravantes, vol à main armée, même des délits sexuels dans des cas exceptionnels). Environ 20 à $50 \%$ des mineurs en démarche de médiation sont des récidivistes. Certains mineurs ou de jeunes adultes participent même plus d'une fois aux projets.

L'expérience a révélé la possibilité de traiter un grand éventail de délits. En plus du vol et de dommages à la propriété, les infractions ayant entraîné des blessures physiques, les menaces, les insultes, l'exercice de la contrainte, la fraude, le détournement de fonds et la falsification de documents sont traités fréquemment. D’après les expériences menées dans le cadre des tribunaux de la jeunesse, jusqu'à $10 \%$ des cas pris en charge par le ministère public ou $30 \%$ de ceux pris en charge par les services d'assistance du tribunal peuvent être traités par voie de médiation (Schrekling et al., 1991 : 33 ; Hartmann, 1995 : 211). Le fait d'inclure d'autres types de compensation peut considérablement augmenter les possibilités de réparation dans le cadre de la justice des mineurs et de la justice criminelle.

\section{Évaluation des projets de médiation}

Les rapports des projets-pilotes à Brunswick, Cologne, Reutlingen, Munich et Landshut sont limités à l'évaluation statistique des cas traités dans les projets. Par conséquent, les données tendent à négliger la question de la sélection des cas et ne permettent pas la comparaison avec d'autres types de prises en charge ou d'autres sanctions. Une recherche

9. Voir Rössner et Bannenberg, (1994:69); dans l'enquête nationale mentionnée précédemment, portant sur plus de 1800 cas de médiation effectués au cours de l'année $1995,9 \%$ concernaient des délits de vol à main armée, $1 \%$ des délits d'ordre sexuel, voir Hartmann et Stroetzel, dans Dölling et al., 1998 : 160-162. 
comparative récente sur les sanctions ${ }^{10}$ indique que l'effet de prévention particulière ou générale produit par la médiation est au moins aussi élevé que celui obtenu avec des sanctions traditionnelles. Certains rapports indiquent même un taux réduit de récidive ${ }^{11}$. En termes d'efficacité interne du projet (c'est-à-dire la capacité de prédiction et l'acceptation de la médiation), les résultats étaient généralement positifs en Allemagne. Ainsi, 80 à $90 \%$ des contrevenants ou victimes approchés par les médiateurs ont accepté cette démarche (Hartmann, 1995 : 212-246 ; Dölling et Henninger, dans Dölling et al., 1998 : 369). Des arrangements ont été conclus dans 67 à $81 \%$ de tous les cas, soit une moyenne de $75 \%{ }^{12}$. La même proportion de contrevenants a tenu ses engagements ${ }^{13}$.

Les délits commis à la fois par les mineurs et les adultes sont en premier lieu les dommages physiques, le vol et dommages à la propriété et, exceptionnellement, des actes criminels comme les vols à main armée. Les données statistiques disponibles pour environ 42 projets de médiation au cours de l'année 1995 révèlent une proportion de $64 \%$ de contrevenants inculpés pour de sérieux dommages physiques, $11 \%$ pour des dommages à la propriété et $9 \%$ pour des vols à main armée (voir Hartmann et Stroetzel, dans Dölling et al., 1998 : 185). Les personnes impliquées en sont en général à leur premier délit. La proportion de récidivistes va de $21 \%$ à Landshut à $48 \%$ à Cologne (Schreckling et al., 1991 : 36). L'enquête de 1995 révèle que $30 \%$ des mineurs et $44 \%$ des adultes contrevenants (âgés de plus de 21 ans) avaient des dossiers criminels antérieurs. En Allemagne, les données relatives au degré de satisfaction des contrevenants et des victimes par rapport aux résultats de la médiation ne sont cependant pas toujours représentatives. Par exemple, la constatation que les deux tiers de ceux qui ont été interrogés étaient satisfaits révèle peu de choses en ce qui concerne les procédés de sélection. Les victimes désirent savoir si les médiateurs sont favorables aux contrevenants ou neutres (Schreckling et al., 1991). Cet intérêt souligne l'importance de placer l'organisation de la

Io. Pour des informations de base sur ce sujet, voir Albrecht, Dünkel et Spiess, 1981 ; Dünkel, 1990b : 553 ss ; Goldblatt et Lewis, 1998 ; Sherman et al., 1998.

II. Dünkel, 1990b ; les études anglo-américaines considèrent en général la réparation comme une sanction, cependant, il y a peu de recherches faites à l'étranger sur l'évaluation comparative des programmes de médiation ; Weigend, 1989 ; Trenczek, 1996, sur le système aux États-Unis ; pour une comparaison internationale des projets de médiation, voir Dünkel, 1990a ; Messmer et Otto, 1992.

I2. Voir Rössner et Bannenberg, 1994 : 69 ; Schreckling et al., 1991 ; Pfeiffer, 1992.

I3. Voir Dünkel et Merigeau, 1990 ; Rössner et Bannenberg, 1994; Hartmann et Stroetzel, dans Dölling et al., 1998 : 188 ss. 
médiation à la fois sous la responsabilité des tribunaux pour mineurs et pour adultes (danger de focaliser les démarches sur le contrevenant) et sous la responsabilité de services privés spécialement destinés à la médiation.

On considère que le projet a réussi quand un accord a été établi hors du tribunal et correspond aux objectifs poursuivis par la démarche de médiation, ce qui arrive dans 70 à $90 \%$ des cas (Schreckling et al., 1991: 44 ; 88 \% dans l'enquête de 1995, voir Hartmann et Stroetzel, dans Dölling et al., 1998 : 185). Si les résultats de la médiation sont positifs, le ministère public ou le juge abandonnent souvent les poursuites ; d'après l'enquête de 1995, c'était le cas dans $86 \%$ de toutes les affaires (voir Hartmann et Stroetzel, dans Dölling et al., 1998 : 193). Quand les poursuites n'ont pas été abandonnées (à cause de la gravité du crime), la sentence était néanmoins réduite (par exemple, commuée en probation ou en d'autres sanctions communautaires mineures).

Tout en tenant compte des expériences favorables et des cas réglés, beaucoup de questions méthodologiques concernant la recherche évaluative restent en suspens. Vu les dépenses considérables consenties pour établir les programmes de médiation, les possibilités de développer de tels projets sont assez limitées (Dölling, 1992 ; Kaiser, 1996 : 1062). Cependant, les démarches de réparation n'exigent pas nécessairement une procédure coûteuse comme l'est la rencontre personnelle entre la victime et son agresseur. Bien souvent, une simple lettre d'excuses ou le remboursement des pertes suffisent. La réparation peut ainsi avoir plus de valeur en tant que sanction indépendante obtenue sans recours à la médiation ( $\S \S 15$ de la loi allemande JGG).

À présent, la plupart des projets de médiation couvrent un maximum de 5 à $10 \%$ (souvent moins) de toutes les affaires relatives aux jeunes contrevenants (Dünkel et Merigeau, 1990: 114). Des estimations confirmées indiquent que plus de $25 \%$ des délits traduits en justice dans le cadre des tribunaux de la jeunesse peuvent faire l'objet de médiation ${ }^{14}$, ce qui constitue « un vaste réservoir » de possibilités. L'étude de Hartmann (1998) dans le champ du droit criminel pour adultes a révélé une proportion de $16 \%$ des cas pouvant être admis en médiation (selon les critères mentionnés plus haut).

I4. Voir Dünkel et Merigeau (1990 : 114); Schreckling et al., (1991 : 33); Pfeiffer (1992 : 342) ; Bannenberg (1993 : 158) ; Wandrey et Weitekamp, dans Dölling et al., (1998 : 142 ss). 
T A B LEA U 1

Programmes de médiation fournis par les organisations locales de protection de la jeunesse et/ou par des organisations privées - comparaison entre les " anciens » et les « nouveaux » États fédéraux en Allemagne (1994).

\begin{tabular}{|l|c|c|c|c|c|c|c|c|c|}
\hline État fédéral & $\begin{array}{c}\text { Départements } \\
\text { de la } \\
\text { protection de } \\
\text { la jeunesse }\end{array}$ & $\begin{array}{c}\text { Médiation par } \\
\text { des } \\
\text { organismes } \\
\text { locaux pour la } \\
\text { jeunesse }\end{array}$ & $\begin{array}{c}\text { Médiation par } \\
\text { des } \\
\text { organisations } \\
\text { privées } \\
\text { seulement }\end{array}$ & $\begin{array}{c}\text { Médiation par } \\
\text { des } \\
\text { organisations } \\
\text { privées et des } \\
\text { organismes } \\
\text { locaux }\end{array}$ & $\begin{array}{c}\text { Total de la } \\
\text { médiation } \\
\text { disponible }\end{array}$ \\
\cline { 2 - 11 } & $\mathbf{N}=100 \%$ & $\mathbf{n}$ & $\%$ & $\mathbf{n}$ & $\%$ & $\mathbf{n}$ & $\%$ & $\mathbf{n}$ & $\%$ \\
\hline $\begin{array}{l}\text { Anciens } \\
\text { États } \\
\text { fédéraux } \\
\text { Total }\end{array}$ & 479 & 230 & 48 & 70 & 15 & 36 & 8 & 336 & 70 \\
\hline $\begin{array}{l}\text { Nouveaux } \\
\text { États } \\
\text { fédéraux } \\
\text { Total }\end{array}$ & 127 & 63 & 49 & 18 & 14 & 31 & 24 & 112 & 88 \\
\hline $\begin{array}{l}\text { Allemagne } \\
\text { Total }\end{array}$ & 606 & 293 & 48 & 88 & 15 & 67 & 11 & 448 & 74 \\
\hline
\end{tabular}

\section{Les résultats d'une enquête nationale}

L'enquête menée en 1994 auprès des départements de protection de la jeunesse et des services privés d'assistance aux mineurs a révélé que la médiation était offerte pratiquement partout dans les anciens États de l'Allemagne et particulièrement dans les nouveaux États fédéraux où $88 \%$ des départements de protection de la jeunesse ont des projets de médiation (voir le tableau 1 ; au sujet des efforts menés en faveur d'une mise en place nationale, voir Kerner et Hassemer, 1994 ; Marks et Meyer, 1994 ; Wandrey et Weitekamp, dans Dölling et al., 1998).

L'étude menée par l'Université de Greifswald ne concernait pas en premier lieu la médiation mais était plutôt centrée sur l'ensemble des nouvelles sanctions de type éducatif (c'est-à-dire les programmes d'acquisition de compétences psychosociales, les mesures de surveillance, les travaux communautaires et la médiation) qui devinrent des éléments de la nouvelle loi de 1990 (JGG). Ces mesures devaient réduire considérablement les sanctions visant l'enfermement, comme la détention dans des centres de détention pour jeunes (Jugendarrest) ou la 
détention dans une institution pour jeunes délinquants (Jugendstrafe $)^{15}$. Cependant, ces tendances nouvelles en faveur de mesures plus éducatives n'ont eu que des résultats modestes. La plupart des départements de protection de la jeunesse ne règlent au cours d'une année qu'un petit nombre de cas au moyen de la médiation ${ }^{16}$. Et encore, dans ces cas là, la médiation est plus considérée comme une mesure particulière, employée occasionnellement ( $a d$ hoc) que comme un programme établi. Pour l'année 1993, les chiffres indiquent que la moitié des départements de protection de la jeunesse ayant pratiqué la médiation ne sont parvenus qu'à régler huit conflits dans les anciens États fédéraux et sept dans les nouveaux États fédéraux. $75 \%$ des départements de protection de la jeunesse n'ont pas traité plus de quinze ou seize cas par année (voir le tableau 2).

Nous avons voulu savoir quel était le nombre de départements de protection de la jeunesse qui offraient la médiation, en utilisant leurs propes ressources ou en faisant appel à des services privés. Sur l'ensemble des questionnaires envoyés, nous avons pu analyser les informations de 531 districts (soit $85 \%$ de tous les départements). Parmi ces districts, $76 \%$ offraient la médiation. Cependant, les départements ne choisissaient cette option que dans $16 \%$ des cas. Les nouveaux États fédéraux, où $88 \%$ des districts proposent des programmes de médiation et ont recours à cette option dans $20 \%$ des cas, soutiennent favorablement la comparaison avec les anciens États fédéraux (où les taux correspondants sont respectivement de $73 \%$ et de $15 \%$ ).

Un projet est considéré comme «bien implanté » s'il rencontre l'une des trois conditions suivantes :

- il permet de traiter annuellement trente cas ;

- il constitue un lieu privilégié de spécialisation pour les travailleurs sociaux (par exemple, un travailleur qui aurait suivi une formation en résolution de conflits, formation prodiguée par l'Association allemande

I5. Les projets en faveur de nouvelles sanctions communautaires de type éducatif sont traités dans le cadre de groupes de travail au niveau des États et dans un groupe de travail fédéral qui permet l'échange régulier d'expériences. Pour un inventaire des projets individuels incluant une brève description, voir Bundesarbeitsgemeinschaft für ambulante Massnabmen nach dem Jugendrecht in der DVJJ (1992).

I6. Un résultat similaire fut obtenu dans l'étude de Wandrey et Weitekamp, dans Dölling et al. (1998 : 133 ss.) : $38 \%$ des projets ne traitaient pas plus de dix cas par an, tandis que $11 \%$ seulement traitaient plus de 100 cas. Beaucoup de départements de protection de la jeunesse qui pratiquent la médiation comme une mesure ad boc dans quelques cas individuels ne mentionnent même pas leurs chiffres. 
TA BLEA U 2

Participants à la médiation (1991-1993). Comparaison des programmes de médiation entre les « anciens » et les « nouveaux » États fédéraux

\begin{tabular}{|c|c|c|c|c|c|c|c|c|c|}
\hline & \multicolumn{3}{|c|}{1991} & \multicolumn{3}{|c|}{1992} & \multicolumn{3}{|c|}{1993} \\
\hline & \begin{tabular}{|c|}
$25 \%$ \\
$n=$
\end{tabular} & $\begin{array}{c}50 \% \\
\mathrm{n}=\end{array}$ & $\begin{array}{c}75 \% \\
\mathrm{n}=\end{array}$ & $\begin{array}{c}25 \% \\
\mathrm{n}=\end{array}$ & $\begin{array}{l}50 \% \\
\mathrm{n}=\end{array}$ & $\begin{array}{c}75 \% \\
\mathrm{n}=\end{array}$ & $\begin{array}{c}25 \% \\
\mathrm{n}=\end{array}$ & $\begin{array}{c}50 \% \\
\mathrm{n}=\end{array}$ & $\begin{array}{c}75 \% \\
\mathrm{n}=\end{array}$ \\
\hline $\begin{array}{l}\text { Anciens États } \\
\text { fédéraux }\end{array}$ & 3 & 7 & 17 & 3 & 8 & 17 & 4 & 8 & 20 \\
\hline $\begin{array}{l}\text { Départements de } \\
\text { la protection de la } \\
\text { jeunesse, }\end{array}$ & \multicolumn{3}{|c|}{128} & \multicolumn{3}{|c|}{178} & \multicolumn{3}{|c|}{210} \\
\hline Cas de médiation & \multicolumn{3}{|c|}{1735} & \multicolumn{3}{|c|}{2574} & \multicolumn{3}{|c|}{3346} \\
\hline $\begin{array}{l}\text { Nouveaux États } \\
\text { fédéraux }\end{array}$ & 1 & 3 & 8 & 2 & 6 & 12 & 3 & 8 & 20 \\
\hline $\begin{array}{l}\text { Départements de } \\
\text { la protection de la } \\
\text { jeunesse, }\end{array}$ & \multicolumn{3}{|c|}{40} & \multicolumn{3}{|c|}{72} & \multicolumn{3}{|c|}{107} \\
\hline Cas de médiation, & \multicolumn{3}{|c|}{338} & \multicolumn{3}{|c|}{785} & \multicolumn{3}{|c|}{1836} \\
\hline Allemagne Total & 2 & 5 & 14 & 3 & 7 & 15 & 4 & 7 & 20 \\
\hline $\begin{array}{l}\text { Départements de } \\
\text { la protection de la } \\
\text { jeunesse, }\end{array}$ & \multicolumn{3}{|c|}{168} & \multicolumn{3}{|c|}{250} & \multicolumn{3}{|c|}{317} \\
\hline $\begin{array}{l}\text { Cas de médiation, } \\
\mathrm{n}=\end{array}$ & \multicolumn{3}{|c|}{2073} & \multicolumn{3}{|c|}{3359} & \multicolumn{3}{|c|}{5182} \\
\hline
\end{tabular}

d'aide à la probation ou d'autres associations de ce genre) ;

- il a initié la création d'un fonds pour les victimes.

Les 61 projets «bien implantés » dans les anciens États fédéraux se situaient principalement en Basse-Saxe, à Hambourg, en Bade-Würtemberg et à Berlin. En Allemagne de l'Est, la plupart étaient situés à BerlinEst et en Saxe. L'étude de ces projets établis montre bien que la médiation est devenue une pratique spécialisée à laquelle les départements de protection de la jeunesse recourent de plus en plus souvent dans pratiquement tous les États fédéraux (voir les résultats d'une autre enquête nationale, Hartmann et Stroetzel, dans Dölling et al. (1998 : 154 ss.).

Durant les quinze dernières années, les sanctions pénales se sont profondément modifiées dans le cadre de la justice des mineurs; ce changement est manifeste quand on considère l'émergence des nouvelles sanctions de type éducatif. On constate aussi une augmentation du recours aux mesures alternatives (sanctions informelles ou diversion) : de $44 \%$ des délits punissables par la loi en 1981 à $68 \%$ en 
F I G URE 1

Sanctions pénales pratiquées dans le système de justice des mineurs en Allemagne 1981-1996

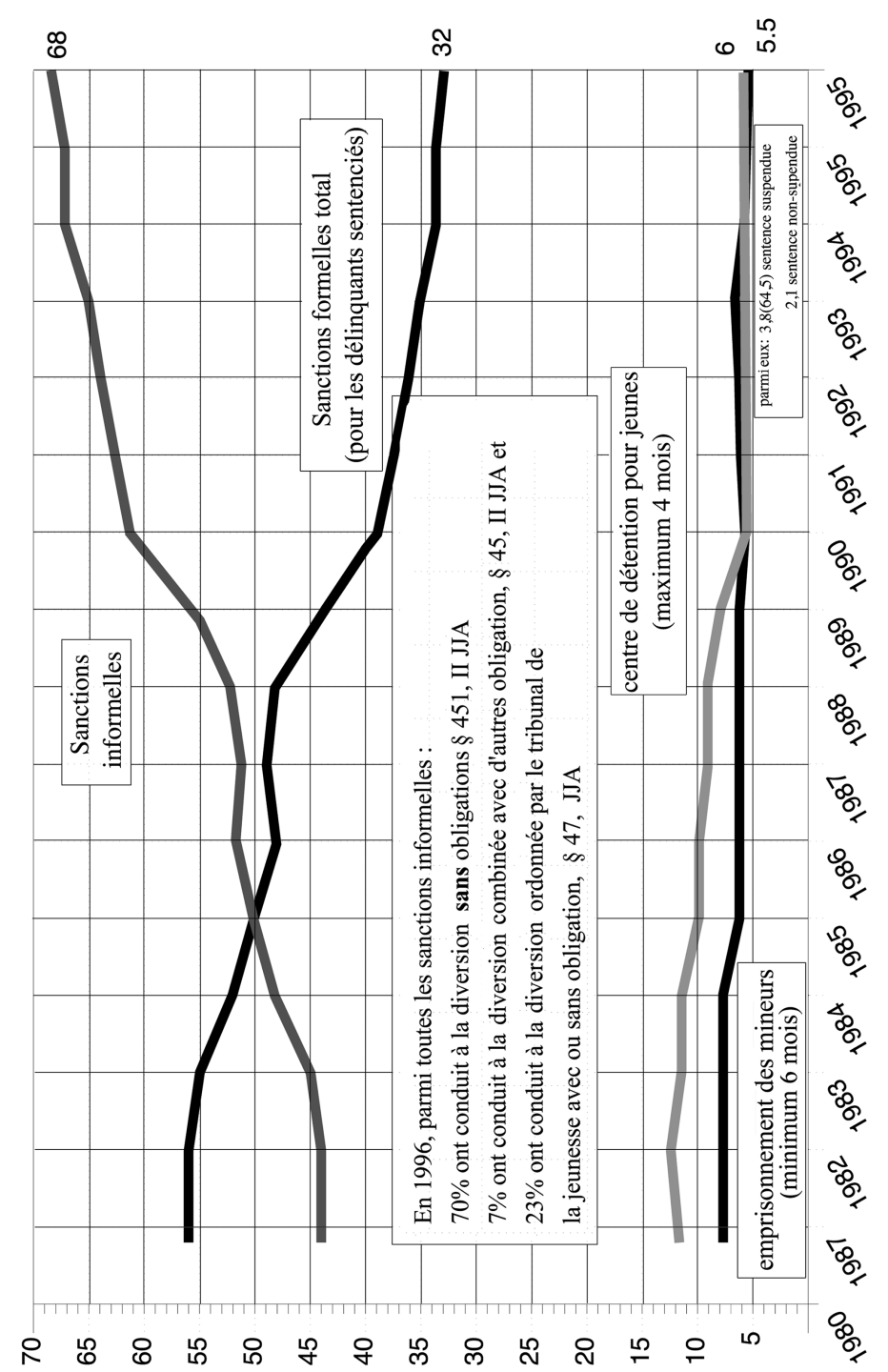

(\%) әธерұนәวגnod 
1996 (Heinz, 1997 et figure 1). Parallèlement, les sentences imposant l'arrestation des mineurs (ce qui veut dire la détention pour quatre semaines maximum dans une institution spéciale) ont diminué de plus de $40 \%$, baissant de $11,4 \%$ à $6 \%$. D'autre part, le nombre de jeunes détenus dans une institution pour mineurs est resté relativement stable, ce qui n'est pas étonnant si l'on tient compte de l'augmentation des sentences reliées à des actes de violence ou à des délits reliés à la drogue. Cette augmentation transparaît clairement dans les sanctions infligées par le tribunal ; on y trouve des mesures purement réformatrices et des travaux communautaires considérés comme des mesures disciplinaires. $34 \%$ des jeunes délinquants enfermés en 1996 ont reçu une ordonnance de travaux communautaires. Cette information coïncide avec les résultats de notre propre enquête auprès des départements de protection de la jeunesse. Cette enquête révèle que les travaux communautaires sont la sanction la plus utilisée parmi toutes les nouvelles sanctions de type éducatif. Le recours à la probation (la sentence suspendue pour une durée maximum de deux ans suivant $\S \S 21 \mathrm{JGG}$ ) a aussi plus que doublé depuis 1965. Malheureusement, les statistiques officielles relatives aux poursuites faites au criminel ne fournissent aucune donnée sur la fréquence des médiations, spécialement en ce qui concerne le rôle du ministère public dans les sanctions alternatives «informelles ». On dispose néanmoins de traces qui indiquent qu'à peine plus de $5 \%$ des délits poursuivis le sont au moyen de la médiation.

\section{Conclusion}

En conclusion, la comparaison des législations, expériences et pratiques européennes ${ }^{17}$ permettent de relever que :

1. Dans le droit criminel, on trouve des principes favorisant le rétablissement de la paix. La médiation et la réparation relèvent de ces principes qui, comme le modèle réhabilitatif, ont été longtemps négligés en faveur d'un modèle plus punitif.

2. La médiation et la réparation sont des réponses appropriées et réalistes qui permettent d'atténuer et même d'éliminer — dans le cas de crimes de gravité mineure ou modérée - le recours à des sanctions imposées par l'État.

I7. Voir à ce sujet Dünkel et Rössner (1989) ; Dünkel (1990a) ; Eser et Walther (1996). 
3. La réparation est un élément essentiel de la pratique juridique en matière de droit criminel dans la plupart des pays européens. La probation ou la réparation sont considérées dans certains pays comme des sanctions indépendantes. Il est clair que la pratique judiciaire a peu recours aux mesures de réparation prévues par le droit criminel. Il serait donc souhaitable que l'on en fasse un plus grand usage.

4. En ce qui concerne les crimes peu ou modérément graves (spécialement les délits tels que les dommages à la propriété et les infractions ayant entraîné des blessures légères), la réparation devrait suivre une procédure de réparation ou de réconciliation indépendante du domaine judiciaire. L'objectif serait de parvenir à un accord sur les modes de réparation entre la partie lésée et l'agresseur. Le règlement extrajudiciaire du conflit met en lumière les raisons principales du désaccord entre parties, il permet aussi aux profanes et aux personnes travaillant dans les services d'aide aux contrevenants ou dans les services de probation d'exercer des activités significatives dans ce domaine. Les personnes impliquées dans la médiation ont néanmoins besoin d'être formées. De telles activités préjudiciaires devraient être considérées comme des moyens de décriminalisation. L'abandon de mesures punitives et répressives ne compromet pas nécessairement les intérêts de la société et la réhabilitation de la victime ou de l'agresseur. Dans le cas de criminalité occasionnelle, on pourrait lever les sanctions si la partie incriminée manifeste des remords sincères.

5. Comme c'est le cas en Angleterre, au Pays de Galles, en Écosse et en Allemagne, la justice pénale des mineurs et des adultes de 14 à 21 ans devrait donner à la réparation le statut de sanction criminelle indépendante. Dans le droit criminel, le processus de réparation va plus loin que la réparation des dommages proposée par le droit civil. Cette démarche suppose que le contrevenant reconnait le mal causé par son action, qu'il est conscient de sa responsabilité sociale et qu'il se conforme aux valeurs et aux normes dominantes. Tout en considérant la réparation comme une sanction, les travaux communautaires devraient aussi constituer une alternative positive pour les contrevenants sans ressources. En tant que sanction criminelle et en vertu des principes de culpabilité et de proportionnalité, la réparation exigée (tout comme les travaux communautaires) ne devraient pas dépasser les sanctions (sous forme d'amendes) qui pourraient être imposées autrement.

6. Le recours à la réparation en tant que sanction exclusive doit pouvoir satisfaire les besoins de la victime. Dans des cas plus graves (par 
exemple des cas de violence), la réparation n'offre pas de garanties préventives suffisantes pour satisfaire aux exigences de la loi et pour rétablir l'ordre social. Cette condition s'applique aux crimes très violents et aux meurtres. Dans ces cas, la médiation pourrait cependant se révéler fort utile pour régler des conflits personnels sous-jacents. Tout effort de réparation manifesté par l'agresseur devrait être matière à une réduction de peine (éventuellement une réduction spéciale). Par ailleurs, les initiatives prises en faveur du développement de la réparation dans le droit criminel ne devraient pas gêner l'exercice du droit à une défense pleine et entière pour l'accusé.

7. Les projets de médiation sont d'abord destinés aux cas relevant du droit des mineurs ou aux cas de délinquance juvénile. Au cours des années 1970, des projets-pilotes analogues se sont développés au Canada et aux États-Unis tandis qu'en Europe, la tendance ne s'est manifestée qu'au cours des années 1980. Des projets de médiation furent alors implantés en Angleterre et au Pays de Galles, en Allemagne, en Autriche, en Norvège ; des projets individuels furent aussi mis en place en France et en Finlande. En Belgique et aux Pays-Bas, cependant, les programmes d'aide aux victimes relèvent d'une sphère d'activité distincte de celle qui concerne la médiation offerte aux jeunes contrevenants par des associations d'assistance et de probation. Actuellement, les projets de médiation sont répartis assez largement dans certains pays comme l'Allemagne, l'Autriche et la Norvège. Le nombre de cas traités est néanmoins plutôt bas en comparaison avec les cas faisant (pour la première fois) l'objet de poursuites. La médiation s'est développée ensuite dans le cadre de la justice criminelle adulte (en Allemagne et en Autriche).

8. Les projets de médiation ont clairement démontré que :

- les victimes acceptent en majorité les offres de réparation, les excuses et considèrent souvent le plein remboursement des pertes matérielles comme un élément secondaire de la démarche ;

- les contrevenants se plient généralement aux accords établis lors de la médiation;

- le taux de succès des programmes de médiation est plus élevé quand les projets sont gérés par des personnes spécialisées et bien formées et quand ces personnes s'investissent à fond dans les procédures de médiation (à l'opposé de la médiation conçue comme une mesure « accessoire » par rapport aux démarches "normales» centrées sur le contrevenant, la médiation ne s'appliquant alors qu'à un nombre limité de cas par an); 
- les deux parties apprécient en général les contacts victimecontrevenant (allègement de l'hostilité et de la peur de la victime, responsabilisation du contrevenant par rapport aux torts faits à la victime, résolution positive du conflit ou réparation des torts) sauf dans les cas de crimes très graves ou dans les cas de désaccords interpersonnels prolongés ;

- même sans avoir recours aux procédures criminelles officielles, les principes de justice, d'égalité, de protection de la victime (contre des enquêtes inopportunes et contre des mesures faisant pression en faveur de la médiation, etc.) et du contrevenant (contre les aveux forcés, les exigences irrationnelles de la victime, etc.) peuvent être garantis ;

- même dans le cadre d'un procès, la réparation peut être envisagée en tant que sanction indépendante ou en tant que condition de probation tout en respectant le droit à un procès équitable ;

- les modalités de réparation, tout comme les sanctions traditionnelles, doivent être envisagées en tenant compte de leur potentiel réhabilitatif et de leur valeur préventive.

9. Les projets de médiation sont encore soumis à des évaluations générales, du moins en Europe. Il faut noter que le développement des projets de médiation a fait dès le départ l'objet de recherches intensives, surtout en Allemagne, en Angleterre, au Pays de Galles et en Autriche. Les résultats des premières recherches menées en Europe et en Amérique du Nord ont révélé que de tels projets pouvaient résoudre divers types de conflits (mêmes ceux reliés à des crimes graves ou à des récidives). Les enquêtes ont aussi révélé que ces démarches se concluaient de façon satisfaisante tant pour la victime que pour l'agresseur. Diverses études menées aux États-Unis ont révélé les résultats favorables de ces mesures de prévention spécifique lorsqu'on les compare aux sanctions traditionnelles. On constate du moins que le taux de récidive n'est pas plus élevé dans les cas traités par médiation que dans les cas faisant l'objet de mesures de probation ou de détention. Les mêmes constatations s'appliquent pour les travaux communautaires qui deviennent une alternative de plus en plus populaire aux courts termes d'emprisonnement, spécialement dans quelques pays d'Europe (voir à ce sujet les résultats de recherche positifs provenant des Pays-Bas).

10. Les critiques à l'encontre des projets de médiation semblent relever plus volontiers des a priori idéologiques que des données empiriques. Cependant, l'étude de certains cas isolés a mis en évidence des éléments contestables qui doivent être pris en considération. Parmi les points sujets à la critique, relevons notamment la pression indirecte 
exercée en vue de participer aux séances de médiation ou en vue d'accepter les propositions faites au cours de cette démarche. Ce type de problème est plus susceptible d'arriver s'il existe une profonde différence de statut social entre la victime et le contrevenant. Un autre motif de critique est lié au développement des mesures prises par l'État pour forcer à la réparation, par exemple en considérant ces mesures comme des mesures additionnelles plutôt que comme des mesures de substitution (ce qui renforce alors le contrôle social). Enfin, la compensation exigée (qui est souvent minime du point de vue matériel) et les rapports limités qu'entretient le système de médiation avec les organisations œuvrant auprès des contrevenants suscitent aussi des critiques.

Aux États-Unis et en Angleterre, de telles critiques ont souligné l'importance de former adéquatement les médiateurs. Au cours de projets analogues menés en Allemagne, ce sont des travailleurs sociaux spécialement formés ou des psychologues qui exercent cette fonction.

Le fait que la démarche de médiation dépende de la bonne volonté des deux parties et que le contrevenant doive faire des aveux a aussi fait l'objet de critiques. Les expériences faites en Allemagne ont démontré l'inefficacité de sanctions plus graves infligées faute d'aveux ou en cas de refus de participer à la médiation.

11. Aucune restriction ne s'applique quant aux délits pouvant faire l'objet de médiation. Alors qu'au départ les projets de médiation concernaient surtout les blessures physiques, les atteintes à la propriété, les insultes mineures, on remarque qu'au cours des dernières années la médiation fut aussi appliquée dans les cas de crimes plus graves. Les crimes violents comme le vol à main armée ou les crimes ayant entraîné des blessures physiques graves sont de plus en plus souvent traités par voie de médiation. De tels projets ont commencé dans le cadre de programmes de traitement des prisonniers (par exemple en Allemagne, en Angleterre et au Pays de Galles, en Suisse et aux États-Unis). La médiation ne se réduit pas aux crimes commis à l'encontre des individus. Des délits d'ordre plus général comme les crimes contre l'environnement et les crimes contre les biens peuvent aussi faire l'objet de mesures de réparation comme les travaux communautaires. Les délits impliquant un plus grand nombre de parties ne devraient pas non plus être automatiquement exclus sous prétexte que la démarche de médiation pourrait prendre plus de temps et se révéler plus difficile.

12. La recherche criminologique devrait jeter un regard critique sur les résultats des mesures réparatrices pratiquées dans et hors du cadre de la justice criminelle. Elle devrait aussi vérifier dans quelle mesure les 
projets de médiation contribuent réellement à régler les conflits et à réconcilier les parties de façon satisfaisante. De tels projets sont évidemment en opposition avec toute forme de punition jugée trop répressive.

La réparation et la médiation en tant qu'instruments favorisant le dialogue, la réconciliation et le retour à la paix ne pourront trouver une place dans la justice criminelle du XXI ${ }^{\mathrm{e}}$ siècle que si elles s'enracinent solidement dans la théorie et la pratique du droit criminel. Nous l'espérons, les démarches réparatrices entamées par le biais de la médiation deviendront un outil durable permettant aux politiques de gestion de la criminalité de sortir du dilemme répression/prévention tout en ne suscitant pas d'attentes irréalistes. Une fois les dédommagements réglés, il faut reconnaître que, dans la plupart des cas, les contacts victime-contrevenant ne sont ni nécessaires ni souhaitables. Si les dédommagements relèvent du domaine matériel et quantitatif, la médiation individuelle (incluant les contacts directs entre la victime et le contrevenant par le biais de la médiation) se veut plutôt une mesure de type qualitatif.

\section{Références}

Albrecht, H.-J., DÜNKEL, F. et SPIESS, G. (1981), « Empirische Sanktionsforschung und die Begründbarkeit von Kriminalpolitik », Monatsschrift für Kriminologie und Strafrechtsreform, vol. 64, p. 310-326.

BANNENBERG, B. (1993), Wiedergutmachung in der Strafrechtspraxis, Bonn, ForumVerlag.

Bundesarbeitsgemeinschaft für ambulante Maßnahmen nach dem Jugendrecht in der DVJJ (dir.) (1992), Ambulante Maßnabmen und sozialpädagogische Jugendhilfeangebote für junge Straffällige, Bonn, Forum-Verlag.

Dölling, D. (1992), « Der Täter-Opfer-Ausgleich; Möglichkeiten und Grenzen einer neuen kriminalrechtlichen Sanctionsform », Juristenzeitung, vol. 47 , p. 493-499.

Dölling, D., et al. (1998), Täter-Opfer-Ausgleich in Deutschland. Bestandsaufnabme und Perspektiven, Bonn, Forum-Verlag.

DÜNKEL, F. (1985), «Victim Compensation and Offender Restitution in the Federal Republic of Germany - A Western-European Comparative Perspective », International Journal of Comparative and Applied Criminal Justice, vol. 9, p. 29-39.

DÜNKEL, F. (1990a), « Médiation délinquant-victime et réparation des dommages. Nouvelle évolution du droit pénal et de la pratique judiciaire dans une comparaison internationale », in DÜNKEL, F. et ZERMATTEN, J. 
(dir.), Nouvelles tendances dans le droit pénal des mineurs, Friedurg, Max-PlanckInstitut für ausländisches und internationales Strafrecht, p. 1-81.

DÜNKEL, F. (1990b), Freiheitsentzug für junge Rechtsbrecher; Situation und Reform von Jugendstrafe, Jugendstrafvollzug, Jugendarrest und Untersuchungshaft in der Bundesrepublik, Deutschland und im internationalen Vergleich, Bonn, ForumVerlag.

DÜNKEL, F., KIRSTEIN, W. et GENG, B. (1998), Soziale Trainingskurse und neue ambulante Maßnabmen in der Bundesrepublik Deutschland, Bonn, Bundesministerium der Justiz.

DünKel, F. et MerigeaU, M. (1990), «Les expériences de médiation délinquant-victime en République fédérale d'Allemagne », in DÜNKEL, F. et Zermatten, J. (dir.), Nouvelles tendances dans le droit pénal des mineurs, Freiburg, Max-Planck-Institut für ausländisches und internationales Strafrecht, p. 95124.

DÜNKEL, F. et RÖSSNER, D. (1989), « Law and Practice of Victim/Offender Agreements », in Wright, M. et Galaway, B. (dir.), Mediation and Criminal Justice ; Victims, Offenders and Community, London, Sage, p. 152-177.

ESER, A. et WALTHER, S. (dir.) (1996), Wiedergutmachung in Kriminalrecht; internationale Perspektiven (vol. 1), Freiburg, Max-Planck-Institut für ausländisches und internationales Strafrecht.

GoldBLATT et LEWIS (dir.) (1998), Reducing Offending : An Assessment of Research Evidence on Ways of Dealing with Offending Behaviour, London, Home Office.

HARTMAnN, A. (1995), Scblichten oder Richten; der Täter-Opfer-Ausgleich und das (Jugend-) Strafrecht, München, Fink Verlag.

HaRTMAnN, U.I. (1998), Staatsanwaltschaft und Täter-Opfer-Ausgleich, BadenBaden, Nomos-Verlag.

HEINZ, W. (1997), «Deutschland», in DÜNKEL, F. et al. (dir.), Entwicklungstendenzen und Reformstrategien im Jugendstrafrecht im internationalen Vergleich, Bonn, Forum Verlag, p. 3-65.

HERING, R.D. et RÖSSNER, D. (dir.) (1993), Täter-Opfer-Ausgleich im allgemeinen Strafrecht, Bonn, Forum-Verlag.

KAISER, G. (1994), « The Juvenile Justice System : The Case of Germany », Legal Studies Forum, vol. 18, p. 319-347.

KAISER, G. (1996), Kriminologie, Heidelberg, Verlag C.F. Müller.

Kawamura, G. et Schreckling, J. (1990), « Täter-Opfer-Ausgleich Eine professionelle soziale Intervention? Überlegungen zur Arbeitsmethodik vor dem Hintergrund der Waage-Fallpraxis », in MARKS, E. et RÖSSNER, D. (dir.), Täter-Opfer-Ausgleich, Bonn, Forum-Verlag, p. 73-114.

Kerner, H.-J. et Hassemer, E. (dir.) (1994), Täter-Opfer-Ausgleich auf dem Weg zur bundesweiten Anwendung?, Bonn, Forum-Verlag.

KILCHLING, M. (1995), Opferinteressen und Strafverfolgung, Freiburg, Max-PlanckInstitut. 
KuHn, A. et al. (1989), "Tat-Sachen » als Konflikt; Täter-Opfer-Ausgleich in der Jugendstrafrechtspflege; Forschungsbericht zum Modellprojekt "Handschlag", Bonn, Forum-Verlag.

MARKS, E. et MEYER, K. (dir.) (1994), Wiedergutmachung und Strafrechtspraxis, Bonn, Forum-Verlag.

MARKS, E. et RÖSSNER, D. (dir.) (1989), Täter-Opfer-Ausgleich - Vom zwischenmenschlichen Weg zur Wiederherstellung des Rechtsfriedens, Bonn, Forum-Velag.

MESSMER, H. (1996), Unrecbtsaufarbeitung im Täter-Opfer-Ausgleich, Bonn, Forum-Verlag.

Messmer, H. et OTto, H.-U. (dir.) (1992), Restorative Justice on Trial. Pitfalls and Potentials of Victim-Offender Mediation. International Perspectives, Dordrecht, Kluwer (NATO ASI Series).

PfeIfFer, C. (1992), «Täter-Opfer-Ausgleich — das Trojanische Pferd im Strafrecht? », Zeitscbrift für Rechtspolitik, vol. 9, p. 338-345.

PfeIfFer, C. (1994), "Wiedergutmaxchung und Strafe aus der Sicht der Bevoelkerung », in Kerner, H.-J., HASSEMER, E., MARKS, E. et WANDREY, M. (dir.) Taeter-Opfer-Ausgleich - auf dem Weg zur bundesweiten Anwendung?, Bonn, Forum-Velag, p. 91-116.

PfEIFFER, C. (dir) (1997), Täter-Opfer-Ausgleich im Allgemeinen Strafrecht, BadenBaden, Nomos-Verlag.

RÖSSNER, D. et BANNENBERG, B. (1994), « Empirische Ergebnisse zum TäterOpfer-Ausgleich », in KAISER, G. et JEHLE, J.-M. (dir.), Kriminologische Opferforschung (vol. 1), Heidelberg, Kriminalistik Verlag, p. 65-88.

SABROTZKY, M. (1997), «Die Entwicklung der Schlichtungsstellen in den neuen Bundesländern hinsichtlich der Aufgabe der außergerichtlichen Schlichtung von Strafsachen ", in HASSEMER, E., MARKs, E. et MEYER, K. (dir.), Zehn Jabre Täter-Opfer-Ausgleich und Konfliktschlichtung, Bonn, ForumVerlag, p. 144-166.

SCHRECKLING, J. et al. (1991), Bestandsaufnabme zur Praxis des Täter-OpferAusgleichs in der Bundesrepublik Deutscbland, Bonn, Bundesministerium der Justiz.

SESSAR, K. (1992), Wiedergutmachen oder strafen; Einstellungen in der Bevölkerung und in der Justiz, Pfaffenweiler, Centaurus-Verlag.

SESSAR, K. et al. (1986), « Wiedergutmachung als Konfliktregelungsparadigma? » Kriminologisches Journal, vol. 18, p. 86- 104.

SHerman et al. (1998), Preventing Crime: What Works, What Doesn't, What's Promising, National Institute of Justice, Research in Brief, Washington (D.C.).

STEFFENS, R. (sous presse), Wiedergutmachung und Täter-Opfer-Ausgleich im Jugendund Erwachsenenstrafrecht in den neuen Bundesländern, Mönchengladbach, Forum-Verlag. 
TRENCZEK, T. (1996), Restitution - Wiedergutmachung, Schadensersatz oder Strafe? Restitutive Leistungsverpflichtungen im Strafrecht der USA und der Bundesrepublik Deutschland, Baden-Baden, Nomos-Verlag.

Voss, M. (1989), « Anzeigemotive, Verfahrenserwartungen und die Bereitschaft von Geschaedigten zur informellen Konfliktregelung », Monatsschrift für Kriminologie und Strafrechtsreform, $\mathrm{n}^{\circ} 72$, p. 34-51.

WeIGEND, T. (1989), Deliktsopfer und Strafverfabren, Berlin, Duncker \& Humblot. 